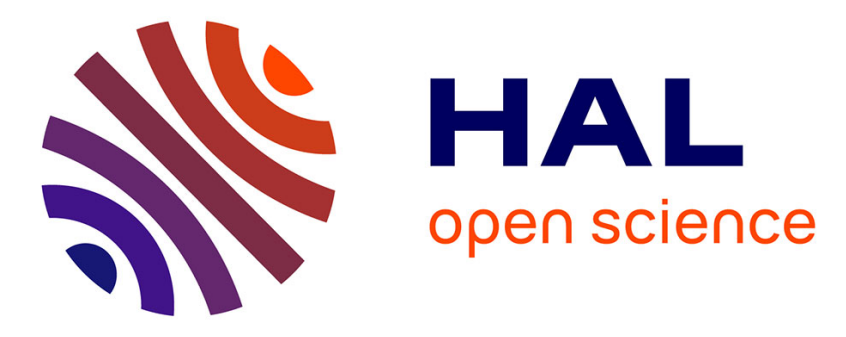

\title{
LTE-M adaptive eNodeB for emergency scenarios
}

Ahmad Hani El Fawal, Ali Mansour, Mohamad Najem, Frédéric Le Roy, Denis Le Jeune

\section{To cite this version:}

Ahmad Hani El Fawal, Ali Mansour, Mohamad Najem, Frédéric Le Roy, Denis Le Jeune. LTE$\mathrm{M}$ adaptive eNodeB for emergency scenarios. ICTC: Information and Communication Technology Convergence, Oct 2017, Jeju Island, South Korea. 10.1109/ICTC.2017.8191035 hal-01707168

\section{HAL Id: hal-01707168 https://hal.science/hal-01707168}

Submitted on 12 Feb 2018

HAL is a multi-disciplinary open access archive for the deposit and dissemination of scientific research documents, whether they are published or not. The documents may come from teaching and research institutions in France or abroad, or from public or private research centers.
L'archive ouverte pluridisciplinaire $\mathbf{H A L}$, est destinée au dépôt et à la diffusion de documents scientifiques de niveau recherche, publiés ou non, émanant des établissements d'enseignement et de recherche français ou étrangers, des laboratoires publics ou privés. 


\section{LTE-M adaptive eNodeB for emergency scenarios}

Conference Paper · October 2017

DOI: $10.1109 /$ ICTC.2017.8191035

CITATIONS

0

5 authors, including:

Ahmad Hani El Fawal

ENSTA Bretagne

2 PUBLICATIONS 1 CITATION

SEE PROFILE

Mohamad Najem

ENSTA Bretagne

14 PUBLICATIONS 9 CITATIONS

SEE PROFILE
READS

310

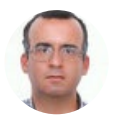

Ali Mansour

ENSTA Bretagne

218 PUBLICATIONS $\quad$ 1,942 CITATIONS

SEE PROFILE

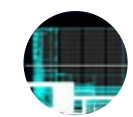

Frederic Le Roy

ENSTA Bretagne

24 PUBLICATIONS 21 CITATIONS

SEE PROFILE

Some of the authors of this publication are also working on these related projects:

Project

Stochastic Signals View project

Project

Ecosystem Monitoring \& Port Surveillance View project

All content following this page was uploaded by Ahmad Hani El Fawal on 28 August 2017. 


\title{
LTE-M Adaptive eNodeB for Emergency Scenarios
}

\author{
A. H. El Fawal ${ }^{(1,2)}$, A. Mansour ${ }^{(1)}$, M. Najem ${ }^{(1)}$, F. Le Roy ${ }^{(1)}$ and D. Le Jeune ${ }^{(1)}$ \\ ahmad.el-fawal@ensta-bretagne.org; mansour@ieee.org; mohamad.najem@ensta-bretagne.fr; \\ frederic.le_roy@ensta-bretagne.fr; denis.le_jeune@ensta-bretagne.fr \\ (1) Lab-STICC, ENSTA Bretagne, 29806 Brest, France; (2) AUL University, Beirut, Lebanon
}

\begin{abstract}
The next generation of mobile systems must provide a balancing strategy towards M2M (Machine-to-Machine) traffic, while maintaining a sustainable Quality of Service $($ QoS $)$ for H2H (Human-to-Human) traffic, especially with the expected exponential growth of the number of M2M devices in the coming years carried by the advance of the IoT (Internet of Things) technology. In normal situations, it is obvious that using a 1.4 MHz bandwidth in Long Term Evolution for Machines (LTE-M), helps in improving M2M device complexity, cost and battery life effectively. However, in emergency scenarios, an expected M2M storm will lead inevitably to a fast resource depletion accompanied by an eNodeB congestion in a split second. In this manuscript, we propose a novel scheme "LTE-M Adaptive eNodeB", which gradually solves the eNodeB overload problem, while keeping the $\mathrm{H} 2 \mathrm{H}$ traffic QoS not to be affected badly. Moreover, We adaptively manage network resources to allow both traffic to efficiently access the LTE network via SimuLTE opensource modeler. Eventually, an evaluation of the mutual impact of $\mathrm{M} 2 \mathrm{M}$ and $\mathrm{H} 2 \mathrm{H}$ coexistence is also presented.
\end{abstract}

Keywords - IoT, LTE-A, LTE-M, NB-IoT, LPWAN, M2M, H2H, SimuLTE, eNodeB, Overload Congestion Mechanism.

\section{INTRODUCTION}

Machine-to-Machine (M2M) communications have increasingly become an attractive area for both research and industry. It is a novel communication technology whereby a large number of "connected devices" can exchange information and perform actions without any direct human intervention. The creativity of this new era is boundless with novel potentials. The future M2M devices should sense and communicate via Internet of Things (IoT) technology, command and control applications in a universal ecosystem network making the human's life much easier. According to Ericsson predictions [1], 28 billion connected devices should be introduced by 2021, and 15 billion of them are expected to use M2M communications.

Although, M2M devices transmit small-sized packages in different time intervals, but due to their specificity and functionality they send their payloads in form of synchronized storms, unlike traditional Human-to-Human $(\mathrm{H} 2 \mathrm{H})$ communications. Therefore, one of the prominent challenges that will face the mobile operators is the fast saturation of the limited bandwidth of Long Term Evolution for Machines (LTE-M) with the rise of M2M devices especially in many emergency cases in which all these devices are requesting to send their data simultaneously (e.g., terrorist attacks, tsunamis, power outages etc.).

The saturation problem leads inevitably to a remarkable impact on both M2M and $\mathrm{H} 2 \mathrm{H}$ traffics, services and appli- cations. According to Heavy Reading [2], mobile operators are spending 20 billion dollars a year to overcome network outages and service degradation.

In this manuscript, we propose a novel scheme "LTEM Adaptive eNodeB" (A-eNB) to address this challenge. Our proposed "A-eNB" solves the M2M overload congestion gradually, while keeping the Quality of Service (QoS) of the $\mathrm{H} 2 \mathrm{H}$ traffic within the acceptable standards. The network adaptation is provided through a dynamic LTE-M bandwidth re-allocation with the objective of maximizing the number of $\mathrm{M} 2 \mathrm{M}$ connections and minimizing the impact on the $\mathrm{H} 2 \mathrm{H}$ traffic.

The main contributions of this paper are the following:

- A new adaptive eNodeB "A-eNB" architecture for LTEM networks.

- Our new model is constructed by many simulations using SimuLTE open-source modeler.

- An evaluation of the proposed A-eNB on both $\mathrm{H} 2 \mathrm{H}$ and M2M traffics.

\section{STATE OF THE ART}

In the upcoming years, a ubiquitous coverage, a long battery life, a low device and deployment cost, and a massive number of devices support are mandatory to satisfy the IoT requirements.

To reach this goal, 3GPP adopts its legacy LTE-A technology by proposing an enhanced version called "LTE-A Pro" that supports the narrow-band machine type communications in two featured technologies: LTE-M and NB-IoT [3]: (i) "Enhanced Machine Type Communication" (eMTC) also known as Long Term Evolution for Machines (LTE-M), which reduces the bandwidth to $1.4 \mathrm{MHz}$ using only 6 Physical Resource Blocks (PRB)s, and (ii) "Narrow Band for IoT" (NB-IoT) which reduces the bandwidth to $180 \mathrm{KHz}$ using only 1 PRB. These two solutions adopt all their specifications based on the IoT requirements, trying to seize the maximum portion of the market.

"LTE-A Pro" new technologies have competitive advantages of using the same hardware and a share spectrum by making them compatible with the legacy LTE-A. In the near future, "LTE-A Pro" technologies are expected to attract a huge amount of future IoT market if it can offer a better IoT platform by allowing customers to scale and manage their business requirements more efficiently.

In [4], the authors concluded that the reduced bandwidth allows for a substantial complexity reduction $81 \%$ but the fast 
depletion of the bandwidth when facing an expected M2M storm in an emergency scenario wasn't discussed. Moreover, in [5], a cross-layer solution was proposed to increase the number of devices that can be served by one eNodeB. The solution combines reduction of the TCP/IP overhead with buffering and clustering concepts in order to maximize efficiency of the transmission of small payloads by a high number of devices. Although, the proposal enables to serve up to $65 \mathrm{~K}$ devices by one eNodeB in case of a $10 \mathrm{MHz}$ bandwidth, but it didn't show the cost on both $\mathrm{M} 2 \mathrm{M}$ and $\mathrm{H} 2 \mathrm{H}$ traffics in a congested eNodeB. Another approach was proposed in [6], where a source modelling was proposed based on Coupled Markov Modulated Poisson Processes (CMMPP) to overcome the massive number of devices problem. Although, the proposed model demonstrates the parallel deployment of $30 \mathrm{~K} \mathrm{M} 2 \mathrm{M}$ devices with reasonable efforts, but it couldn't scale to the $52 \mathrm{~K}$ $\mathrm{M} 2 \mathrm{M}$ devices recommended in the IoT requirements. In [7], despite the mathematical model for LTE downlink bandwidth allocation that was proposed with the aim of providing a good QoS for each UE, the coexistence between LTE-M and LTEA systems and the bandwidth adaptation are not spotted. In [8], a proposed cognitive-based radio access strategy with a priority queuing scheme is applied in LTE-A networks with $\mathrm{M} 2 \mathrm{M} / \mathrm{H} 2 \mathrm{H}$ coexistence distinguishing $\mathrm{M} 2 \mathrm{M}$ devices based on their traffic QoS requirements. Although an analytical model is developed in normal scenarios, but the expected surge number of M2M devices which might have higher priority during disaster scenarios and how to deal with this sticky situation hadn't been discussed.

To sum up, in the literature, many approaches are trying to find an ideal solution for the massive access problem and its consequences. But, it can be noticed that the existing architectures provide only preliminary solutions, without any projection to the expected exponential growth of M2M storms especially in disaster scenarios. To the best of our knowledge, our adaptive solution is the first solution to address this problem from its roots in a smart, adaptive and robust methodology, which can result to a "Clean LTE Traffic" free from any M2M congestion.

\section{LTE-A AND LTE-M DATA-RATE}

In order to study the LTE-A and LTE-M bandwidths and their limitations, especially the mutual influence in between $\mathrm{H} 2 \mathrm{H}$ and $\mathrm{M} 2 \mathrm{M}$ traffics one towards the other, we introduce first the time-frequency resources and the correlation with the data-rates for both $\mathrm{H} 2 \mathrm{H}$ and $\mathrm{M} 2 \mathrm{M}$ traffics.

In LTE-A, time-frequency resources are subdivided as shown in Fig. 1:

In the time domain, the largest unit of time is the radio frame $(10 \mathrm{~ms})$, which is composed of ten sub-frames (1 $\mathrm{ms})$. Each sub-frame has two slots (0.5 ms each slot). Each slot comprises seven Orthogonal Frequency Division Multiple Access (OFDMA) symbols (0.5/7 ms)[9].

In the frequency domain, resources are grouped into units, such that one unit of:

a) One sub-carrier (a small channel spaced at $15 \mathrm{KHz}$ with the adjacent channel) for a duration of one OFDMA symbol is termed as a Resource Element (RE) with a $15 \mathrm{KHz}$ spacing.

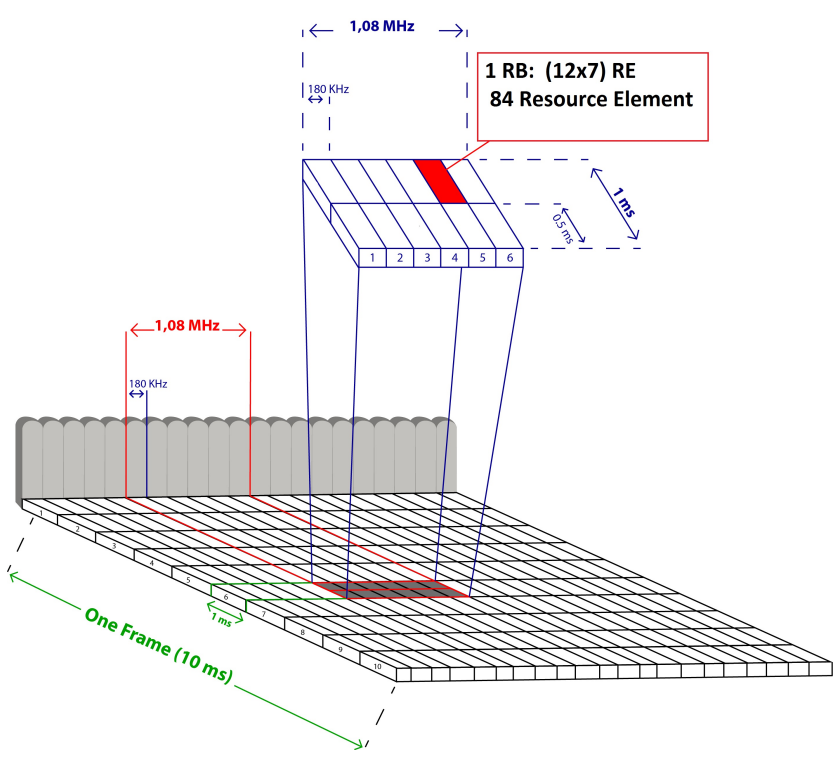

Fig. 1. BL limitation for LTE-M carrier within LTE-A carrier.

b) 12 sub-carriers for a duration of one slot is termed a Resource Block (RB) with a $180 \mathrm{KHz}$ bandwidth.

c) 12 sub-carriers for a duration of one sub-frame is termed a PRB with $180 \mathrm{KHz}$ bandwidth. Thus, a RB comprises $7 \times 12$ $=84$ REs, while a PRB comprises $7 \times 12 \times 2=168$ REs. As a result, a PRB is the minimal unit that can be scheduled for one UE.

With the expected high number of devices per cell (more than $52 \mathrm{~K}$ ), we study the maximum data-rate in a classical LTE-A and LTE-M:

a) In LTE-A, each RE can transmit 6 bits in the best modulation scheme (64QAM). Consequently, the maximum data-rate can be calculated as follows:

$$
\begin{array}{r}
\text { Rate }_{\text {Max }}=N_{\text {sub-carriers }} \times N_{O F D M A} \times N_{\text {slots }} \\
\times N_{P R B} \times N_{\text {bits } / R E}
\end{array}
$$

Let's assume that we have a $20 \mathrm{MHz}$ total bandwidth (with a single antenna). As one PRB is equivalent to $180 \mathrm{KHz}$, then there are $100 \mathrm{PRBs}$ available with $2 \mathrm{MHz}$ guard-band in 20 $\mathrm{MHz}$ total bandwidth. So, the maximum data-rate for the 100 PRBs is about 100800 bits/ms (100 Mbps approx.).

b) The previously computed maximum data-rate is usually dedicated to $\mathrm{H} 2 \mathrm{H}$ users. However, LTE-M technology dedicates in the 3GPP Rel-13, a $1.4 \mathrm{MHz}$ of the total bandwidth for M2M communications. Following the same previous calculation but with 6 PRBs, QPSK modulation and half-duplex mode; the maximum data-rate is reduced to $1 \mathrm{Mbps}$ in up-link (UL) and $1 \mathrm{Mbps}$ in down-link (DL) for M2M traffic.

As a result, the $1 \mathrm{Mbps}$ data-rate is not enough, especially in disaster storms (shown in section V). Therefore, the available bandwidth will suffer from a huge degradation, requiring an efficient solution to tackle this challenge.

\section{LTE-M ADAPTIVE ENODEB}

As previously explained, the bandwidth dedicated for $\mathrm{M} 2 \mathrm{M}$ is denoted hereafter as 'Bandwidth-Limited' (BL). The size of 
the BL defines the number of M2M devices that are enabled to send their data simultaneously to the eNodeB.

The main goal of this study is to allow both M2M and $\mathrm{H} 2 \mathrm{H}$ traffics to access the network resources efficiently in both normal and emergency events. To this end, we propose a promising approach, which extends the classical functionality of the eNodeB and includes an adaptive control of the bandwidth based on the M2M load status throughout the network. The proposed Adaptive eNodeB (A-eNB) manages automatically both $\mathrm{H} 2 \mathrm{H}$ and $\mathrm{M} 2 \mathrm{M}$ traffics. Based on the instantaneous requests of $\mathrm{M} 2 \mathrm{M}$, a part of the total bandwidth will be dedicated to fulfill these sudden needs.

To this end, our proposed Adaptive eNodeB for LTE-M networks, depicted in Fig. 2, operates at different conditions depending on the M2M load state throughout the network:

\section{A) Initial State "A-eNB-INI"}

In normal situations, the A-eNB works similar to any legacy eNodeB in LTE-A/LTE-M networks. A part of the total bandwidth is dedicated for LTE-M traffic. For instance, a total of 20 $\mathrm{MHz}$ (100 PRBs) will be divided into M2M bandwidth with a basic Bandwidth-Limited (BL0 $=1.4 \mathrm{MHz}$ ) by reserving 6 PRBs for M2M devices, and the remaining bandwidth (94 PRBs) are dedicated for $\mathrm{H} 2 \mathrm{H}$ devices, as represented in Fig. 3.

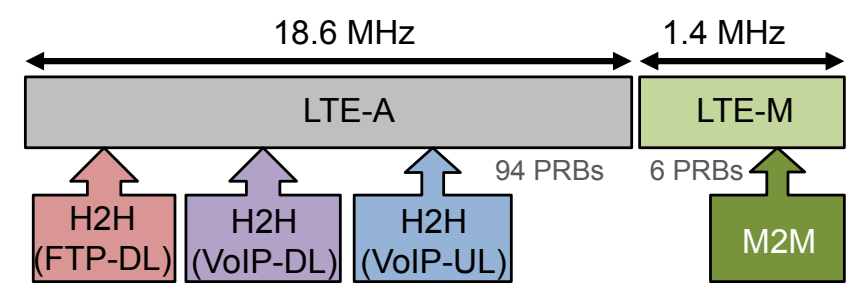

Fig. 3. Initial State "A-eNB-INI" (BLO = 1.4 MHz).

\section{B) Emergency States "A-eNB-EMG[1]" to "A-eNB-EMG[M]"}

In the case of a disaster, a huge number of M2M devices saturate the initial LTE-M bandwidth (BL0) by their storm briefly. When the available resources reach threshold $(0)$, the A-eNB increases its bandwidth from BL0 to BL1 $=2.8 \mathrm{MHz}$ to allow more M2M devices accessing the network. Similarly, if the available resources reach the next threshold again caused by an additional M2M storm, the "A-eNB" adapts gradually BL1 till it reaches $\mathrm{BL}(\mathrm{M})=[(\mathrm{M}+1) * \mathrm{BL} 0] \mathrm{MHz}$, as shown in Fig. 4.

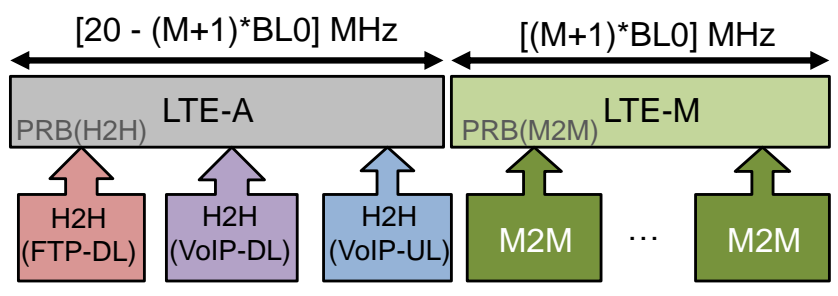

Fig. 4. "A-eNB-EMG(M)"/BL(M).

For a given stage $\mathrm{M}$, we can calculate:

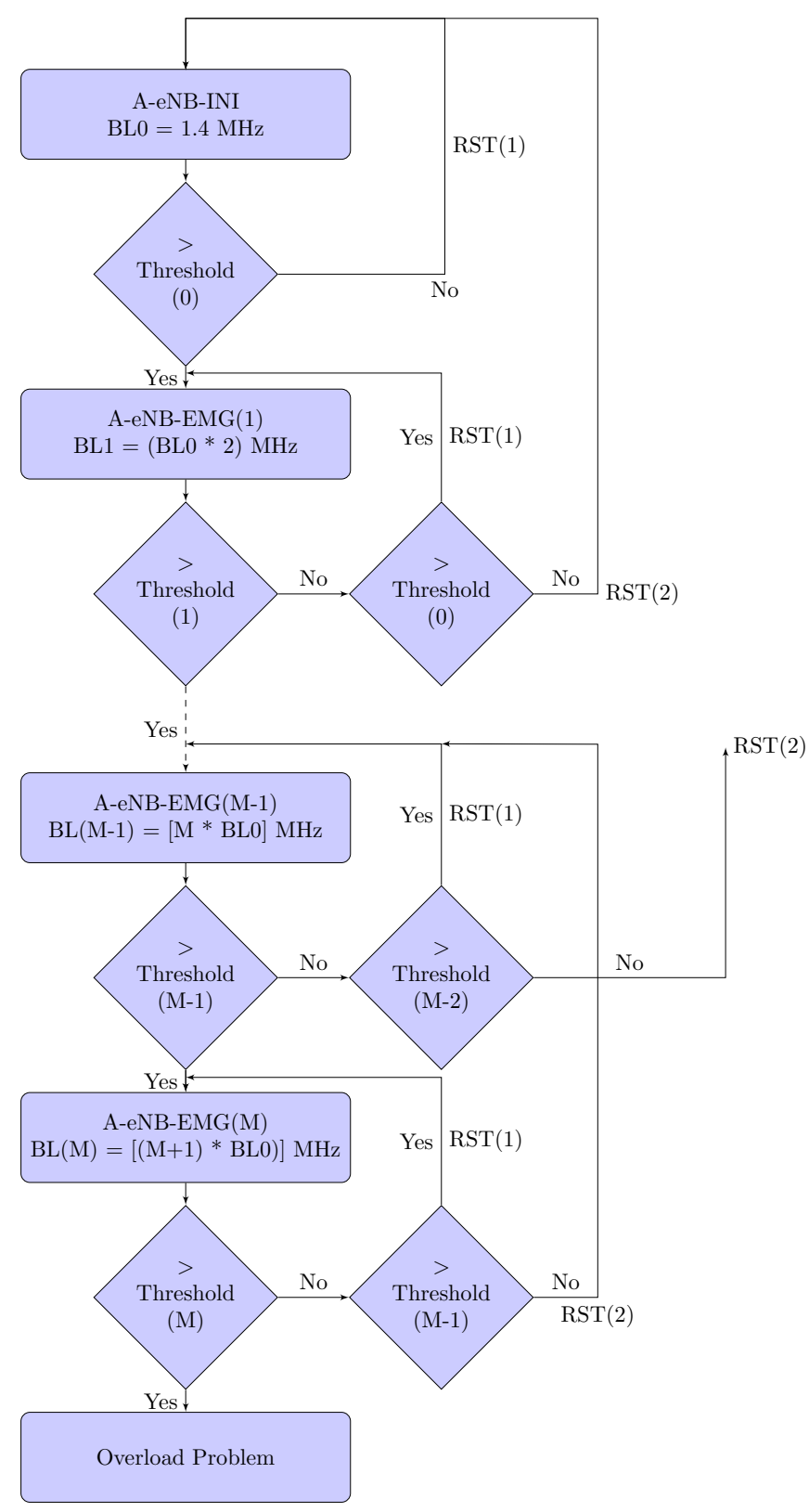

Fig. 2. Flow Chart for the LTE-M Adaptive eNodeB

- The total number of PRBs reserved for M2M devices: $\mathrm{PRB}(\mathrm{M} 2 \mathrm{M})=(\mathrm{M}+1) * 6$.

- The maximum M2M data-rate:

$$
\begin{aligned}
\text { Rate }_{\text {Max }}=N_{\text {sub-carriers }} & \times N_{O F D M A} \times N_{\text {slots }} \\
& \times N_{P R B(M 2 M)} \times N_{\text {bits } / R E}
\end{aligned}
$$

- The total number of PRBs reserved for $\mathrm{H} 2 \mathrm{H}$ devices: $\mathrm{PRB}(\mathrm{H} 2 \mathrm{H})=100-(\mathrm{M}+1) * 6$ 
- The maximum $\mathrm{H} 2 \mathrm{H}$ data-rate:

$$
\begin{aligned}
\text { Rate }_{\text {Max }}=N_{\text {sub-carriers }} \times & N_{O F D M A} \times N_{\text {slots }} \\
& \times N_{P R B(H 2 H)} \times N_{\text {bits } / R E}
\end{aligned}
$$

Once the number of M2M connections starts decreasing, the "A-eNB" reduces iteratively the LTE-M bandwidth either one step back "RST(1)" or two steps back "RST(2)" based on the current threshold until achieving the initial state at the end of the disastrous events, and consequently resumes operating similar to any legacy e-NodeB.

Following this methodology, the "A-eNB" has the smartness, adaptability and robustness to lend progressively a temporary bandwidth up to $\mathrm{BL}(\mathrm{M})$ of the total $\mathrm{H} 2 \mathrm{H}$ bandwidth to $\mathrm{M} 2 \mathrm{M}$ devices tentative use which soak up inevitably any $\mathrm{M} 2 \mathrm{M}$ storm with a minimum impact on $\mathrm{H} 2 \mathrm{H}$ devices.

\section{CASE STUdy}

In real life, the emergency events such as terror attacks, natural disasters and multiple accidents are not predictable at all. With vague scenarios and lack of statistics and researches about the behavior of M2M devices throughout emergency scenarios, we try in this section to build a case study based on some use-cases and 3GPP technical reports.

In [10], a use-case, in which the LTE-M technology is expected to fulfill the M2M requests efficiently with a cutoff point of $80 \mathrm{~K}$ devices per sector for an interval of 4 upload and 4 download transfers per day (with full security) in normal scenarios.

Supporting more than $52 \mathrm{~K} \mathrm{M} 2 \mathrm{M}$ devices per cell is one of the LTE-M targets in order to scale to the IoT requirements [11]. This is the reason why, we consider in our use-case three different groups selected according to the parameters set to different models proposed by 3GPP GERAN TR [12] as follows:

a) Group1 contains 20K Environmental monitoring devices, which send 200 Bytes with a rate of 1 message per hour.

b) Group 2 consists of $20 \mathrm{~K}$ Assisted Medical devices, which dispatch 100 Bytes with a rate of 8 messages per day.

c) Group 3 contains $20 \mathrm{~K}$ Asset tracking devices, which send 50 Bytes with a rate of 100 messages per day.

By analyzing the behavior of M2M devices in the normal and disaster situations, we realize that M2M devices send their payloads with a data-rate equal to:

[(4800 Bytes/86400 sec * 20K devices $)+(800$ Bytes/86400

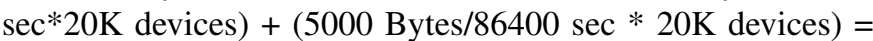
2453 Bytes per second $* 8=0.02$ Mbps.

Consequently, If we compare it with the maximum data-rate in LTE-M (1 Mbps), we conclude that in ideal cases, LTE-M can work efficiently without any congestion problem.

But actually, in real scenarios, the M2M devices send their payloads in a synchronize manner. So, we may expect to have an enormous M2M traffic in a split second even during normal cases in form of three M2M group types (20K M2M devices each). We assume that in each group, $20 \%$ of M2M devices will be synchronized to send their payloads simultaneously. Consequently, we might expect 5 normal storms received from each 5 M2M sub-category (4000 M2M devices for each). As a result, it is expected to receive different storms from each group in different interval as follows:

a) Group1-Storm: The first group sends its payload (200 Bytes) with a rate of 1 message per hour. Consequently, the total rate is equal to: (200 Bytes * $4 \mathrm{~K}$ devices) per second $=6.4 \mathrm{Mbps}$. Because M2M Group1 devices are sending their payloads with a rate of 1 message per hour, so this type of storms will be repeated $24 \times 20=480$ storm/day.

b) Group2-Storm: following the same equation with a payload $=100$ Bytes, the storm can reach 3.2 Mbps total payload rate. As a result, we expect to receive $8 \times 20=160$ storm/day, if we take into consideration that M2M Group2 devices interval is 8 messages/day.

c) Group3-Storm: similar to the two previous storm calculations, but with 50 Bytes payload, this storm peaks at 1.6 Mbps as total payload rate. If we know that the repetition of group3 devices is 100 messages/day, we conclude that this storm repetition is about $100 \times 20=2000$ storm/day.

In emergency cases, we expect to receive the same 5 different storms from each group similar to the previous storms (Group[1-3]-storms) but with an interval of each second throughout the emergency case.

If we compare the different storms in normal and emergency cases, with the maximum uplink data-rate in LTE-M (1 Mbps), we conclude that the available bandwidth will suffer from a huge degradation in both cases.

As a conclusion, we emphasize the need of a coexistence study on how to allow both $\mathrm{M} 2 \mathrm{M}$ and $\mathrm{H} 2 \mathrm{H}$ traffics to access the network efficiently in both normal and emergency events with a minimal impact one towards the other.

\section{Simulations And Results}

A new eNodeB architecture was proposed in section IV which takes into account disastrous and normal conditions. The purpose of this section is to evaluate the A-eNB by measuring the cost on both $\mathrm{M} 2 \mathrm{M}$ and $\mathrm{H} 2 \mathrm{H}$ traffics.

\section{A. Platform and Simulation Parameters:}

In our previous work [13], different results are concluded according to two different platforms in an emergency event full of $\mathrm{H} 2 \mathrm{H}$ and $\mathrm{M} 2 \mathrm{M}$ devices, which require extra investigation using different parameters among several scenarios and platforms. To this end, we use the open-source network simulator SimuLTE Modeler 0.9.1, in an environment of $O M N e T++4.6$ and INET 2.3.0 on a cluster server, as shown in Fig. 5.

The considered simulation settings are: $200 \mathrm{sec}$ total simulation time, eNodeB-UE max. distance $300 \mathrm{~m}$, terminal velocity of $120 \mathrm{Km} / \mathrm{h}$ with a linear mobility of UEs, total bandwidth $20 \mathrm{MHz}$.

\section{B. Scenarios and Result Discussions:}

In our scenarios, we assume that for:

a- H2H Traffic: The scenario consists of $30 \mathrm{H} 2 \mathrm{H}$ users (10 FTP-DL, 10 VoIP-UL, 10 VoIP-DL) during normal conditions. In an emergency event, additional $90 \mathrm{H} 2 \mathrm{H}$ users start to 


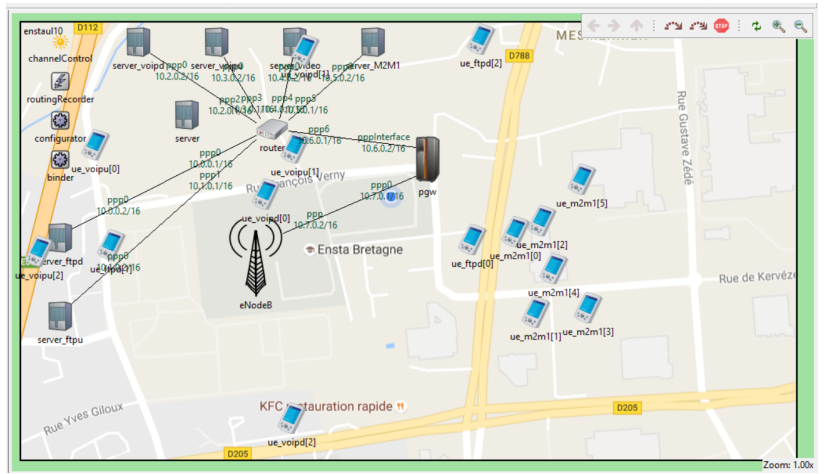

Fig. 5. Architecture with the SimuLTE modeler.

\begin{tabular}{|c|l|l|}
\hline \multirow{4}{*}{ VoIP Model [14] } & Parameter & Settings \\
\cline { 2 - 3 } & Packet Size & 40 Bytes \\
\cline { 2 - 3 } & Interval & $20 \mathrm{~ms}$ \\
\cline { 2 - 3 } & Talkspurts and Silences & Default \\
\hline \multirow{3}{*}{ FTP Model } & Parameter & Settings \\
\cline { 2 - 3 } & Packet Size & 536 Bytes \\
\cline { 2 - 3 } & Interval & $1 \mathrm{sec}$ \\
\cline { 2 - 3 } & File Size & $20 \mathrm{MB}$ \\
\hline
\end{tabular}

TABLE I

H2H TRAFFIC MODELS

operate (30 FTP-DL, 30 VoIP-UL, 30 VoIP-DL) as consequence of the emergency scenario. The $\mathrm{H} 2 \mathrm{H}$ traffic models are represented in Table I.

b- M2M Traffic: The M2M traffic models are mapped to three selected groups of IoT applications according to the parameters set by 3GPP GERAN TR [12] as mentioned in section $\mathrm{V}$.

1) SIM-LTE-A: At first, the impact of $\mathrm{M} 2 \mathrm{M}$ on $\mathrm{H} 2 \mathrm{H}$ traffic in an LTE-A network with a legacy eNB is experimented. To this end, the simulated architecture is composed of: $i$ ) a fixed number of $\mathrm{H} 2 \mathrm{H}$ traffic (40 FTP-DL, 40 VoIP-UL, 40 VoIPDL), and (ii) a variable number of M2M devices [100, 200, 300], connected all together to a legacy eNB.

The measured network performance is depicted in Fig. 6. The VoIP traffic do not reveal a considerable effect of the increasing M2M traffic load within the LTE-A network, as the priority of voice traffic to access the network is higher than the M2M communication. However, the file transfer traffic suffers from a significant degradation of the download performance; for only 300 M2M devices the data downloaded by FTP-DL traffic decreases by $41 \%$ comparing to $100 \mathrm{M} 2 \mathrm{M}$ devices. This is also due to the network priority; FTP has a lower priority than VoIP, and the same priority as M2M traffic which results in a significant congestion when accessing the network.

2) SIM-A-eNB-INI: At this stage, we model the LTE-M network with the aim to measure the improvement on both $\mathrm{M} 2 \mathrm{M}$ and $\mathrm{H} 2 \mathrm{H}$ traffics. To this end, we consider the same traffics as the previous simulation, but with a dedication of 1.4 MHz for M2M devices (BL0 $=1.4 \mathrm{MHz}$ ). The results show that similar to the previous results in "SIM-LTE-A", the VoIP traffic is not affected by M2M traffic, but a significant improvement in the FTP traffic can be noticed as shown in Fig. 8; for example, with $300 \mathrm{M} 2 \mathrm{M}$ devices the FTP traffic has a gain of $82 \%$ comparing to the previous results in "SIM-

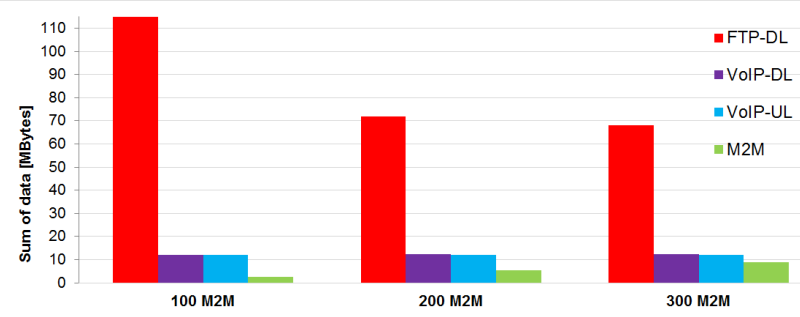

Fig. 6. Impact of M2M traffic on $\mathrm{H} 2 \mathrm{H}$ traffic in a LTE-A legacy scenario.

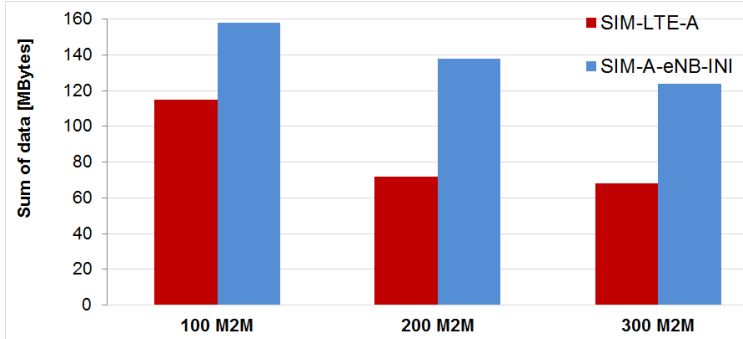

Fig. 7. FTP-DL improvement in SIM-A-eNB-INI comparing to SIM-LTE-A.

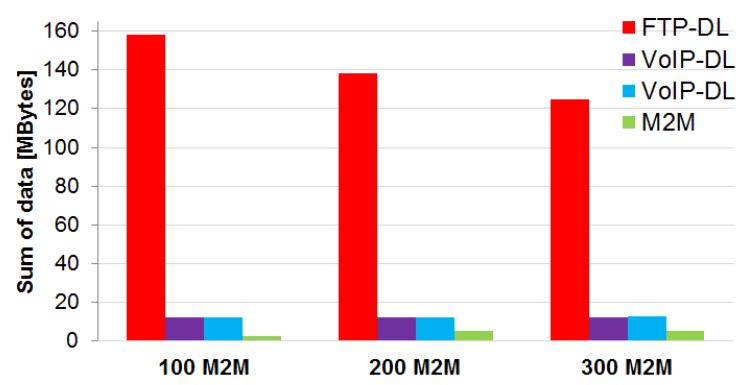

Fig. 8. Improvement on FTP-DL in a LTE-M (BL0=1.4 MHz).

LTE-A" as depicted in Fig. 7.

Meanwhile, the M2M traffic reaches its peak (4.9 MB) starting from $300 \mathrm{M} 2 \mathrm{M}$ devices as depicted in Fig. 8.

Indeed, LTE-M improves the FTP traffic significantly, but a bandwidth saturation problem appears, limiting the number of M2M devices that attempt to access the network.

3) SIM-A-eNB-EMG1: In order to experiment the improvement of our proposed solution on both the FTP traffic and the number of M2M devices accessing the network, we simulate our proposed "A-eNB" in its first act toward an M2M storm by switching from its initial state "SIM-A-eNB-INI" in which $\mathrm{BLO}=1.4 \mathrm{MHz}$ to the first emergency state "SIM-A-eNBEMG1" in which BL1 $=2.8 \mathrm{MHz}$. Then, we evaluate the different traffic performance during this emergency stage. To this end, a fixed number of $\mathrm{H} 2 \mathrm{H}$ traffic is considered (40 FTPDL, 40 VoIP-UL, 40 VoIP-DL) with an increasing number of M2M devices [300, 400, 500, 600, 700], connected all together to the "A-eNB". The network performance are depicted in Fig. 9. 


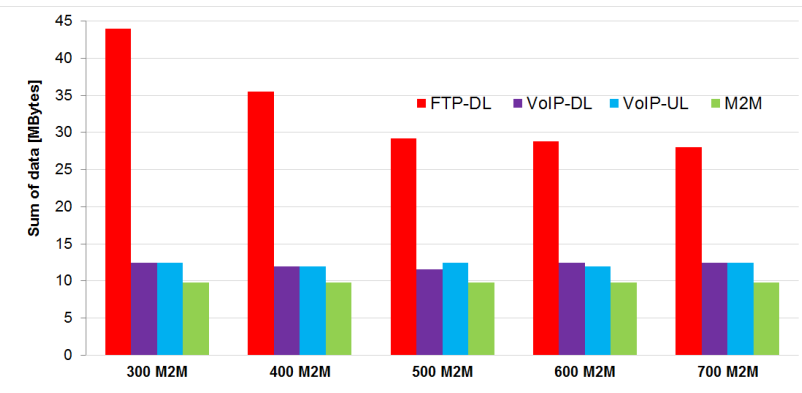

Fig. 9. Improvement on M2M traffic in Emergency State1 "A-eNB-EMG1" (BL1=2.8 MHz).

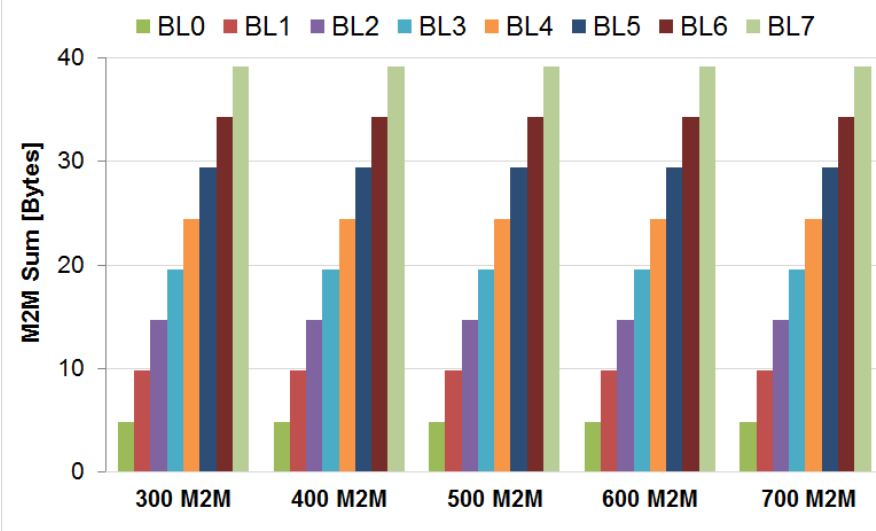

Fig. 10. M2M saturation points comparing to different "A-eNB" states $(M=7)$.

The VoIP traffic is not affected by the increasing M2M traffic, similarly to the previously two scenarios SIM-LTE-A and SIM-A-eNB-INI. We recall that the target is to maximize the number of M2M devices accessing the network and to minimize the impact on FTP traffic. In this emergency stage, the M2M traffic is significantly improved, comparing to SIMA-eNB-INI results (e.g., in $300 \mathrm{M} 2 \mathrm{M}$ devices the improvement is $100 \%$ ). Meanwhile $36 \%$ of FTP traffic is affected, if we compare the FTP traffic in $300 \mathrm{M} 2 \mathrm{M}$ devices to the FTP traffic in $700 \mathrm{M} 2 \mathrm{M}$ devices. This is due to the new bandwidth allocation, deduction of $1.4 \mathrm{MHz}$ from $\mathrm{H} 2 \mathrm{H}$ to $\mathrm{M} 2 \mathrm{M}$ bandwidth (BL1 $=2.8 \mathrm{MHz}$ ). It is important to recall that this is a temporary degradation during the emergency event only, which worth the FTP traffic sacrifice toward the M2M traffic gain as we can realize in the next paragraph. The M2M bandwidth reaches its cut-off point (9.8 MB) starting from 300 M2M devices because of the saturation of BL1, which requires additional actions from the "A-eNB" to absorb the increasing M2M storm.

4) Predictive-SIM-A-eNB-EMG2-(M)": In this predictive scenario we estimate the expected actions taken by the "AeNB" toward a continuous escalated storm by switching from its first emergency state "SIM-A-eNB-EMG1" till it reaches "SIM-A-eNB-EMG(M)", by increasing BL1 $=2.8 \mathrm{MHz}$ till $\mathrm{BL}(\mathrm{M})=[(\mathrm{M}+1) * \mathrm{BL} 0)] \mathrm{MHz}$, we estimate a gain $=\mathrm{M}$ in the M2M traffic comparing to SIM-A-eNB-INI results as depicted in Fig. 10 (suppose $M=7$ ).
Also, we expect a stability in the VoIP traffic with good QoS and a minimum FTP request fulfillment.

To sum up, it is clear that the proposed "A-eNB" in an emergency scenario absorbs gradually the M2M storm while keeping the QoS of $\mathrm{H} 2 \mathrm{H}$ devices within the acceptable standards.

\section{CONCLUSION}

The support of M2M communications in an IoT environment requires a parallel establishment of many new features. In this manuscript, we have proposed an effective solution as an extension to the classic eNodeB in LTE-M networks.

By implementing this novel solution, an adaptive reallocation of the bandwidth leads for an essential resolution for any presumable M2M storm definitely. Based on SimuLTE modeler, the results proof that by leasing some folds of LTE$\mathrm{M}$ classic bandwidth, we can achieve a significant gain in the M2M traffic.

\section{REFERENCES}

[1] ERICSSON. (2015) Ericsson mobility report, on the pulse of the networked society. Accessed: 20-May-2017. [Online]. Available: http://www.ericsson.com/res/docs/2015/mobility-report/ericssonmobility-report-nov-2015.pdf

[2] Heavy Reading (2016), Mobile Network Outages Service Degradations, Survey Analysis. Accessed: 24-June-2017. [Online]. Available: http://www.spirentfederal.com

[3] 3GPP - Release 13 analytical view version Sept. 9th (2015). Accessed: 20-May-2017. [Online]. Available: http://www.3gpp.org/release-13

[4] V. Saxena, J. Bergman, Y. Blankenship, A. Wallen, and H. S. Razaghi, "Reducing the modem complexity and achieving deep coverage in lte for machine-type communications," in 2016 IEEE Global Communications Conference (GLOBECOM), Dec 2016, pp. 1-7.

[5] J. Plachy, Z. Becvar, and E. C. Strinati, "Cross-layer approach enabling communication of high number of devices in 5G mobile networks," in Wireless and Mobile Computing, Networking and Communications (WiMob), 2015 IEEE 11th International Conference on. IEEE, 2015, pp. 809-816.

[6] M. Laner, P. Svoboda, N. Nikaein, and M. Rupp, "Traffic models for machine type communications," in Wireless Communication Systems (ISWCS 2013), Proceedings of the Tenth International Symposium on. VDE, 2013, pp. 1-5.

[7] G. Sergiy, A. D. A. M. Khodayer, and A. J. H. Dheyaa, "Results of development of model for bandwidth management in LTE downlink with Resource Allocation Type 1," in The Experience of Designing and Application of CAD Systems in Microelectronics, Feb 2015, pp. 409413.

[8] S. A. AlQahtani, "Performance analysis of cognitive-based radio resource allocation in multi-channel LTE-A networks with $\mathrm{M} 2 \mathrm{M} / \mathrm{H} 2 \mathrm{H}$ coexistence," IET Communications, vol. 11, no. 5, pp. 655-663, 2017.

[9] S. Sesia, I. Toufik, and M. Baker, LTE-the UMTS Long Term Evolution. Wiley Online Library, 2015.

[10] M. Lauridsen, I. Z. Kovacs, P. Mogensen, M. Sorensen, and S. Holst, "Coverage and Capacity Analysis of LTE-M and NB-IoT in a Rural Area," in 2016 IEEE 84th Vehicular Technology Conference (VTC-Fall), Montreal, Canada, Sept 2016, pp. 1-5.

[11] Y. P. E. Wang, X. Lin, A. Adhikary, A. Grovlen, Y. Sui, Y. Blankenship, J. Bergman, and H. S. Razaghi, "A Primer on 3GPP Narrowband Internet of Things," IEEE Communications Magazine, vol. 55, no. 3, pp. 117123, March 2017.

[12] "3GPP TR 45.820, cellular system support for ultra low complexity and low throughput internet of things," Tech. Rep., August 2016.

[13] A. H. E. Fawal, A. Mansour, F. L. Roy, and D. L. Jeune, "RACH Overload Congestion Mechanism for M2M Communication in LTE-A: Issues and Approaches, Marrakech, Morocco," International Symposium on Networks, Computers and Communication (ISNCC), May 2017.

[14] D.-H. Nguyen, H. Nguyen, and E. Renault, "WE-MQS-VoIP priority: An enhanced LTE downlink scheduler for voice services with the integration of VoIP priority mode," International Journal of Advanced Computer Science and Applications IJACSA, vol. 7, no. 7, pp. 560-567, 2016. 